\title{
The proxy-measurement of intelligence quotients using a relational skills abilities index 访
}

\author{
Dylan Colbert*, Mira Dobutowitsch, Bryan Roche, Caroline Brophy \\ Maynooth University, Ireland
}

\section{A R T I C L E I N F O}

\section{Article history:}

Received 16 July 2016

Received in revised form 16 March 2017

Accepted 20 March 2017

\section{Keywords:}

Relational Frame Theory

Relational skills

Intelligence

IQ

Relational Abilities Index

\begin{abstract}
A B S T R A C T
Relational Frame Theory proposes that levels of sophistication with relational concepts may underlie intellectual performance. In order to further elucidate this relationship, the current study examined correlations between scores on a novel Relational Abilities Index (RAI) and a range of widely-used cognitive ability measures, including Full Scale IQ. In Study 1, 35 adult participants completed a battery of cognitive assessments, comprising of the National Adult Reading Test, the Rey Auditory Verbal Learning Test, the Trail Making Test, the Cognitive Failures Questionnaire and a RAI assessment at two time periods. In Study 2, a full WAIS-III assessment and RAI was administered to 25 college students. Results indicate that performance on the RAI displayed impressive degrees of correlation with the three main IQ indices, three of the four IQ subindices, and three of the four cognitive ability measures, suggesting that the RAI assessment may represent a promising potential proxy measure of Full Scale IQ.
\end{abstract}

(c) 2017 Elsevier Inc. All rights reserved.
Intelligence is commonly believed to lie beyond the remit and descriptive powers of behavior analysis (Abramson, 2013; Block, 1981; Putnam, 1975; Schlinger, 2003). Theoretical objections to hypothetical constructs (Skinner, 1974), a preference for functional (i.e., in terms of environment-behavior relationships) accounts, as well as an apparent difficulty in accounting for the generativity of language and cognition (Hayes, Barnes-Holmes, \& Roche, 2001), are often employed to support the argument that a behavioristic account of intelligence is not only difficult technically, but inappropriate conceptually. However, recent advances in a behavior-analytic account of language and cognition, known as Relational Frame Theory (RFT; Hayes et al., 2001; see also Dymond \& Roche, 2013) have led to new insights into how we might conceive intellectual behavior in a non-mentalistic manner (e.g., Hayes, 1994; O'Hora, Pelaez, \& Barnes-Holmes, 2005; O'Toole, Barnes-Holmes, Murphy, O'Connor, \& Barnes-Holmes, 2009; Smith, Smith, Taylor, \& Hobby, 2005) as well as the development of intervention protocols that have shown early promise in increasing intelligence quotients (Cassidy, Roche, Colbert, Stewart, \& Grey, 2016; Cassidy, Roche, \& Hayes, 2011; Dixon, Whiting, Rowsey, \& Belisly, 2014; Moran, Stewart, McElwee, \& Ming, 2010). As such, RFT-inspired measures are increasingly being looked upon within the behavior-analytic

\footnotetext{
is Declaration of interests: The assessment protocol described in this paper has been developed by Dr. Bryan Roche as part of a commercially available treatment intervention available at RaiseYourlQ.com.

* Corresponding author at: Maynooth University Department of Psychology, National University of Ireland, Maynooth, Co. Kildare, Ireland.

E-mail address: Dylan.colbert.2011@mumail.ie (D. Colbert).
}

community as conceptually-sound proxies for IQ with acceptable construct validity.

Behavior analysts have proposed theoretical objections to essentialistic conceptualization of intelligence, suggesting such accounts commit the logical errors of reification (Gottfredson, 1998; Gould, 1981; Howe, 1990) and circular reasoning (Schlinger, 2003). While a behavior-analytic perspective may propose theoretical objections to the concept of $g$, the practical utility of IQ tests in providing an index of intellectual performance for a given individual in a given assessment, is increasingly being recognised. Indeed, psychometricians have long posited that the term intelligence merely refers to the collection of behaviors that cannot be separated from their context (Schlinger, 2003) and this is an acceptable position for most behavioral researchers concerned about reification. In any case, IQ remains the benchmark for indexing intelligence, and provides the comparative litmus test for any new potential measure of intellectual performance, even where the model of intelligence differs radically from those on which IQ testing is based, as does the current model.

RFT represents the convergence of several decades of research focused on a key skills repertoire known as derived relational responding or relational framing, referred to as a verb rather than a noun, to draw attention to framing as a behavioral skill rather than to frames as mental entities. Relational responding refers to the process of responding to one stimulus in terms of its contextually controlled arbitrary relationship to another. For example, responding to the word "cat" in terms of its relationship to images of cats represents a form of relational responding in terms of stimulus equivalence or coordination. On the other hand, responding to a $5 \mathrm{c}$ coin as worth less than a $10 \mathrm{c}$ coin 
represents a form of relational responding in terms of a relation of comparison (i.e., more/less). It is the presence of contextual cues (e.g., the spoken word "more"), that controls the relational response to any given stimulus.

Relational responding comes in a variety of other forms or 'frames', such as coordination ("cat is the same as kitty"), opposition ("big is opposite to small"), hierarchy ("an apple is a type of fruit"), analogy ("foot is to sock, as hand is to glove"), deixis ("I am here and you are there") and temporality ("morning comes before afternoon"). Importantly, when an individual acquires an understanding of several such relationships, a network of relations between numerous stimuli can be understood, allowing the individual to derive relations between stimuli in the network that have not been explicitly taught. For example, if a child is taught that Jamie is taller than Joanne and Joanne is taller than Aoife, the child can derive that Jamie is therefore taller than Aoife, but only given appropriate training to do so. Hayes et al. (2001) proposed that this learned behavior of deriving relations between and among stimuli is a behavioral process that gives rise to much of human cognition.

RFT suggests that a relatively small variety of relational frames may yield the full array of cognitive skills, like deductive reasoning, problem solving, analogies and language (Barnes-Holmes, Barnes-Holmes, Roche, \& Smeets, 2001; Cassidy et al., 2011, 2016; Hayes \& Stewart, 2016). As such, intelligence is not treated as something one "has", but rather as a generalized set of relational skills (i.e., applicable to any set of stimuli) that are learned, and that therefore could be improved.

\section{The relationship between relational responding and measures of IQ}

Many standard IQ tests contain items that can be understood in terms of relational frames and as tests of derived relational responding (DRR), potentially indicating a degree of overlap between intellectual performance and relational responding proficiency. For example, the Wechsler Vocabulary subtest assesses simple relations of sameness between objects and words, by asking such questions as; "What does simple mean?" or by showing a picture of a carrot and asking; "What is this?". Comparison-based tasks are also commonly assessed by questions such as: "Michelle is 2 years younger than Peter and 5 years older than Sam. If Sam is 6 how old is Michelle?". Relations among relations (analogies) are also widely assessed on standardized IQ tests due to their conceptual relevance to intelligence (Esher, Raven, \& Earl, 1942; Sternberg, 1977; see Cassidy, Roche, \& O'Hora, 2010 for a full conceptual unpacking of IQ test items in terms of relational concepts).

Various correlational analyses have identified the close relationship between relational responding proficiency and performance on a number of IQ indices, subindices and subtests (Dixon et al., 2014; Gore, Barnes-Holmes, \& Murphy, 2010; Moran et al., 2010; O'Hora et al., 2005, 2008; O'Toole et al., 2009). O'Hora et al. (2005) found that performance on a complex relational task involving the derivation of temporal relations, predicted performance on two of the three subtests of the Wechsler Adult Intelligence Scale-III (WAIS-III; Wechsler, 1997) included in the analysis (Vocabulary \& Arithmetic). In a subsequent study, O'Hora et al. (2008) reported that successful completion of a similar task was associated with higher Full Scale and Verbal IQ. Furthermore, O'Toole et al. (2009) reported that performance on a relational task involving temporal and distinction relations predicted scores on the Kaufman Brief Intelligence Test (Kaufman \& Kaufman, 2004). These results were complimented by a later study by Gore et al. (2010) who identified strong correlations between perspective-based relational responding and scores for Full Scale, Verbal and Performance IQ scales of the Wechsler Abbreviated Scale of Intelligence (WASi; Wechsler, 1999) in a sample of adults with intellectual disabilities (see also Dixon et al., 2014). While these correlational analyses alone are not sufficient to support the RFT claim that DRR is foundational to intellectual behavior, they do suggest a degree of functional overlap between the fluency of relational responding and intellectual performance. This functional overlap may allow a relational skills measure to provide an estimate of IQ in certain research contexts in which an estimate is all that is required or in which the researcher or clinician wishes to assess intellectual capacity in purely functional terms.

Cassidy et al. (2011) demonstrated the efficacy of two relational frame multiple exemplar training (MET) interventions in increasing intelligence quotients in samples of children. The MET protocol refers to a technique in which children are posed with a large number of relational "problems" to solve, involving nonsense words and relational cues (e.g., CUG is Opposite to BEH, BEH is Opposite to VEK, Is CUG Opposite to VEK?), all of which take the same form but each of which involves different stimuli. Completion of MET over many sessions and months was correlated with significant rises in Full Scale IQ in both Experiment $1(M=27$ points $)$ and Experiment $2(M=13$ points). In order to assess pre- and post-intervention relational responding proficiency, a preliminary Relational Abilities Index (RAI) was also devised, consisting of 60 relational tasks, such as those outlined above, assessing the relational frames of Same, Opposite, More than and Less than. Significant rises in RAI scores were observed following intervention. Correlations between the RAI score and baseline IQ were not assessed as the RAI was employed only to ensure that relational skills were being increased due to the intervention.

In a follow-up study, Cassidy et al. (2016) reported clinically significant IQ gains following the implementation of a modified version of the MET intervention across two experiments with larger samples and more rigorous controls. In both experiments, a revised RAI consisting of 55 questions was presented pre- and post-intervention. In Experiment $1(n=15)$, RAI scores failed to correlate with baseline IQ scores $(r=0.13)$. However, in Experiment 2, which employed a larger sample $(n=30)$, RAI scores at baseline did correlate significantly with baseline levels of Verbal Reasoning ( $r=0.67)$, Numerical Reasoning $(r=0.43$ ) and overall Educational Aptitude $(r=0.66)$, supporting the RFT-inspired hypothesis that relational skills are closely related to intelligence, with some suggesting that these repertoires are possibly even synonymous (e.g., Hayes \& Stewart, 2016).

\subsection{The current study}

The aim of the current study is to conduct a preliminary assessment of the utility of the Relational Abilities Index (RAI) as a proxy measure of Full Scale IQ and a range of widely used cognitive ability measures. The purpose of using a range of measures that assess different cognitive domains is precisely to begin investigating which aspects of cognitive functioning relational skills best correspond to and to allow for the assessment of both convergent and divergent validity. That said, as the traditional litmus test for assessing the validity of a potential proxy measure of intelligence is an investigation into the strength of its relationship to general intellectual performance, the correlation between Full Scale IQ and RAI score represents the focal point of our analysis.

In Study 1, adult participants completed the National Adult Reading Test (NART; Nelson, 1982), the Rey Auditory Verbal Learning Test (RAVLT; Rey, 1958; English version: Taylor, 1959), the Trail Making Test (TMT; Lezak, 1995) and the Cognitive Failures Questionnaire (CFQ; Broadbent, Cooper, FitzGerald, \& Parkes, 1982), as well as a RAI assessment at two separate time periods for the purpose of assessing testretest reliability. Due to the level of correlation between IQ and the NART (Nelson, 1982), RAVLT (Mitrushina, Boone, Razani, \& D'Elia, 2005) and TMT (Ardila, Pineda, \& Rosselli, 2000), we anticipated significant correlations between performance on these measures and the RAI. Conversely, as the CFQ has not been found to show a strong relationship with IQ (Broadbent et al., 1982), we predicted that the RAI would not correlate with this metric, thereby providing some divergent validity for the RAI. Study 2 focused on the degree of correlation between the RAI and scores on the WAIS-III. It was expected that performance on 
the RAI would correlate significantly with Full Scale IQ as calculated using the WAIS.

\section{Study 1}

\subsection{Method}

\subsubsection{Participants}

Participants ( $n=35,18$ female) comprised of 26 college students and 9 non-students (mean age $=30.6, S D=7.31$ ). The follow-up sample $(n=27,14$ female, mean age $=29, S D=7.96)$ was comprised of 23 college students and 4 non-students, who were re-administered the RAI at follow-up session. An a priori power analysis indicated that a sample size of 24 would be sufficient to establish a correlation coefficient of 0.6 at an alpha of 0.05 . The 0.6 coefficient was chosen because it was the minimally acceptable coefficient necessary to support the convergent validity of the RAI.

\subsubsection{Materials}

2.1.2.1. Relational Abilities Index (RAI). The Relational Abilities Index assessment used here was precisely as employed by Cassidy et al. (2016) and was administered via the public website RaiseYourIQ. com. ${ }^{1}$ The assessment presented participants with 55 syllogistic relational puzzles (see Fig. 1), which required approximately 14 min to complete. It consisted of 29 Same/Opposite tasks and 26 More/Less tasks which progressed in difficulty across trials. A total of 248 stimuli, comprised of three-letter nonsense words (e.g., BEF, DIL, FAS), were used with no stimulus being repeated. Participants were informed in advance that questions would ask them about the relationships between these nonsense words, and that the answer could be arrived at by reading the statements carefully. The questions remained on screen until the user responded but a time limit of $30 \mathrm{~s}$ was applied. The total number of correct responses produced within the $30 \mathrm{~s}$ response window across the 55 trials was taken as the metric of relational skill proficiency. For a full description on the composition and procedural details of the RAI, see Cassidy et al. (2016).

2.1.2.2. National Adult Reading Test (NART). The NART (Nelson, 1982) requires participants to read out a list of words whose pronunciation does not adhere to regular stress and grapheme-phoneme rules. NART performances show moderate to high correlations with general cognitive ability and IQ (Crawford, Parker, Stewart, Besson, \& De Lacey, 1989; Crawford, Stewart, Cochrane, Parker, \& Besson, 1989; Nelson, 1982). Originally standardized against the Wechsler Intelligence Scale (WAIS; Wechsler, 1955), regression equations are available to predict WAIS test scores.

2.1.2.3. Rey Auditory Verbal Learning Test (RAVLT). The RAVLT (Rey, 1958; English version: Taylor, 1959) is a general memory test. Performance on the RAVLT correlates with IQ, learning and executive functioning (Bolla-Wilson \& Bleecker, 1986; Crossen \& Wiens, 1994; Mitrushina et al., 2005). The main metric of interest is the RAVLT 1 to 5 , which refers to the participant's total number of words correctly recalled after five presentations of a 15 -word list.

2.1.2.4. Trail Making Test (TMT). The TMT is used to assess scanning and visuomotor tracking, cognitive flexibility and divided attention (Lezak, 1995). TMT performance is affected by education, intelligence and age (Heaton, Grant, \& Matthews, 1986; Spreen \& Strauss, 1998). The test consists of two parts, requiring participants to draw a line connecting encircled numbers in ascending order in the first part (TMT A) and connecting numbers and letters in part two (TMT B) while being

\footnotetext{
1 This is a commercial website in which the third author has an interest. The authors have declared a potential conflict of interest to the Editor of this journal.
}

timed. Due to differences in compliance to instructions, measures taken often also include the difference in time between TMT A and TMT B (Spreen \& Strauss, 1998).

2.1.2.5. Cognitive Failures Questionnaire (CFQ). The CFQ comprises of 25 items that assess everyday slips and errors in memory and perception, such as failing to notice road signs, forgetting names or bumping into people (Broadbent et al., 1982). Scores on the CFQ correlate with measures of incidental learning, attention and memory, but not IQ (Broadbent et al., 1982; Herrmann, 1982; Martin, 1986; Martin \& Jones, 1983).

\subsubsection{General experimental procedure}

The procedure for this study received ethical approval from Maynooth University's Research Ethics Committee, and was conducted in accordance with the code of ethics of the Psychological Society of Ireland. All participants were tested individually in a quiet room for both assessments. The initial testing period was approximately $40 \mathrm{~min}$ in duration, whereas follow-up RAI assessments required approximately $14 \mathrm{~min}$, and took place 3-4 months following the baseline assessment. Upon entering the experimental setting, participants were informed about the general procedure of the study and signed the consent form. The cognitive measures were administered in a counterbalanced order, with the only exception being that the administration of the RAI always took place during the delay interval for the RAVLT, for the purposes of time efficiency.

\subsection{Results and discussion}

No data was excluded from the following analyses and data was not transformed in any way other than in line with the usual means of calculating scores on the various tests employed. RAI scores ranged from 25 to 52 out of a total of $55(M=41.2, S D=7.51)$. The mean NART Error Score was $19.86(S D=5.76)$, which converts into a mean FSIQ Score of $106.03(S D=7.16)$. Results from a Shapiro-Wilk test indicate that scores for RAI and NART Error measures were normally distributed. Fig. 2 presents histograms and probability plots displaying the distribution of RAI scores NART Error scores.

Time between the RAI test and retest ranged from 105 to 131 days with an average of 116.52 days $(S D=5.35)$. Test-retest reliability for RAI Scores was $0.809(p<0.001)$, with a mean score change ranging from -3 to $+12(M=3.26, S D=4.07)$.

\subsubsection{Correlational analyses}

RAI scores were found to be moderately negatively correlated with NART Error Scores and TMT B-A scores. That is, higher RAI scores were indicative of better performances on the NART and TMT. In addition, RAI scores showed strong positive correlations with scores for the RAVLT. As predicted, RAI scores did not correlate with scores on the CFQ. Table 1 lists correlation coefficient and $p$ values for the main inter-correlations. For the full correlational matrix, see Appendix A.

In summary, Study 1 found that RAI scores showed moderate-tostrong correlations with scores on the NART, RAVLT and TMT but not with the CFQ. These findings support the convergent and divergent validity of the RAI as a measure of intelligence. Test-retest reliability for the RAI was also found to be satisfactory.

\section{Study 2}

Study 2 aimed to extend the findings of Study 1 in investigating how the RAI functions as a measure in comparison to the full WAIS-III IQ test battery using a sample of college students. In effect, this will serve as a sort of experimentum crucis of a novel measure of intelligence insofar as standardized IQ tests provide universally agreed indices of $g$ and any novel test must ultimately speak to such measures, even if only to understand their divergences from them. 
Same/Opposite

VIX is the same as YAR

Is VIX the same as YAR?

\section{YES}

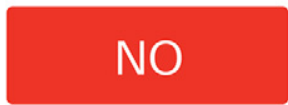

3

$\mathrm{HEH}$ is opposite to WAK

WAK is the same as XIX

Is HEH the same as XIX?
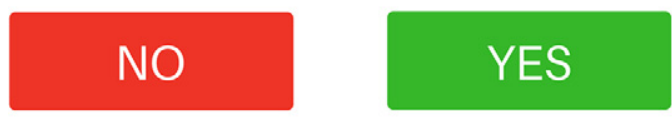

5

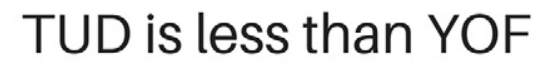

REQ is less than TUD

GAX is less than REQ

Is GAX more than YOF?
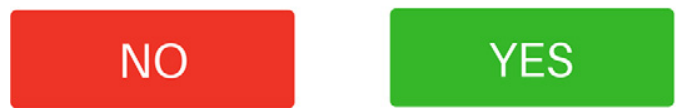

More/Less

2

CUS is more than QEX

Is CUS less than QEX?
4

NON is less than NIG

PIL is less than NON

Is NIG less than NON?
NO
6

NUK is opposite to ZEQ

ZEQ is opposite to BIL

$\mathrm{BIL}$ is the same as BEL

Is NUK opposite to BEL?
NO

Fig. 1. Sample tasks from the RAI assessment

\subsection{Method}

\subsubsection{Participants}

All participants ( $n=25,14$ females) were currently attending third level education or had recently graduated ( $<1$ year). As such, this cohort was not representative of the general population and was sampled ad hoc for reasons of convenience. Participants ranged from 18 to 44 years old $(M=22.2$ years, $S D=5.13)$. An a priori power analysis indicated that a sample size of 24 would be sufficient to establish a correlation coefficient of 0.6 and an alpha of 0.05 .

\subsubsection{Settings and materials}

3.1.2.1. Wechsler Adult Intelligence Scale III (WAIS-III). Each participant was administered the 13 core subtests of WAIS-III (Wechsler, 1997), allowing computation of all seven IQ indices and subindices. These subtests included Picture Completion, Vocabulary, Digit-Symbol Coding, Similarities, Block Design, Arithmetic, Matrix Reasoning, Digit Span, Information, Picture Arrangement, Comprehension, Symbol Search \& Letter-Number Sequencing.

3.1.2.2. Relational Abilities Index (RAI). Participants were also required to complete the RAI assessment to measure their level of proficiency in relational responding, precisely as used in Study 1.

\subsubsection{General experimental procedure}

The procedure for this study received ethical approval from Maynooth University's Research Ethics Committee, and was conducted in accordance with the code of ethics of the Psychological Society of Ireland. Upon entering the private experimental room, participants were informed about the general nature and procedure of the experiment 

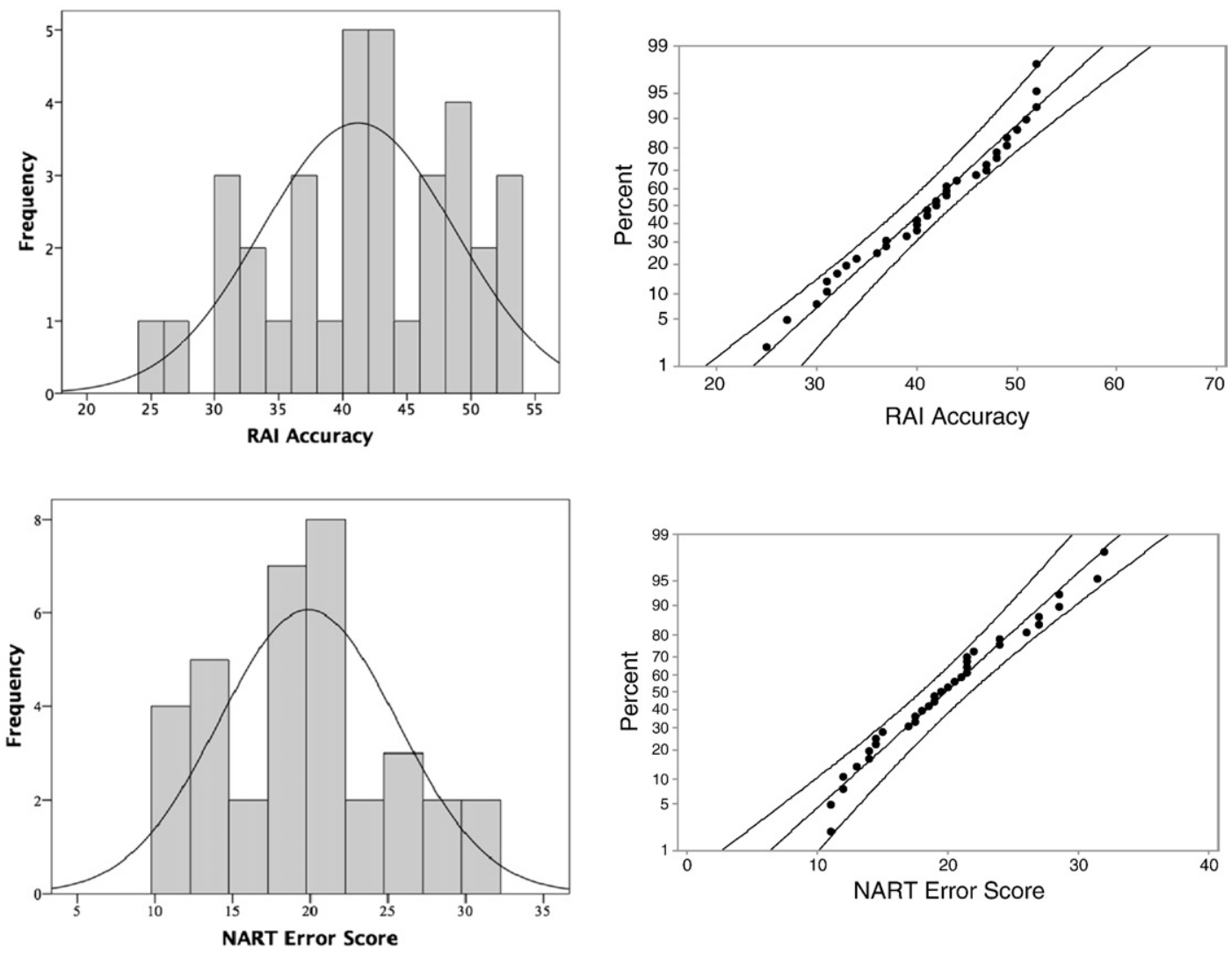

Fig. 2. Histograms (left) and probability plots (right) outlining the distribution of RAI scores and NART Error scores in Study 1.

and signed the consent form. Administration time for the WAIS-III was approximately $85 \mathrm{~min}$.

\subsection{Results and discussion}

No data were excluded from analysis and data were not transformed in any way aside from in line with normal score calculation procedures for the WAIS and RAI (total correct responses). Fig. 3 displays histograms and probability plots which represent the distribution of RAI $(M=48.2)$ and WAIS Full Scale IQ $(M=117.2)$ scores. Results from a Shapiro-Wilk test of normality indicate that RAI scores were not normally distributed $(p=0.003)$.

\subsubsection{Correlational analyses}

Relational Ability Index scores showed moderate-to-strong significant correlations with scores for Full Scale, Verbal and Performance IQ, as well the four IQ subindices (Verbal Comprehension, Working Memory, Perceptual Organisation and Processing Speed). Table 2 outlines the

Table 1

Correlations between RAI score and the battery of cognitive measures.

\begin{tabular}{lll}
\hline & \multicolumn{2}{l}{ RAI } \\
\cline { 2 - 3 } & Coefficient & Sig. level \\
\hline NART $^{\mathrm{a}}$ & $-0.583^{* *}$ & $<0.001$ \\
${\text { RAVLT } 1 \text { to } 5^{\mathrm{a}}}_{\text {TMT B-A }^{\mathrm{b}}}$ & $0.696^{* *}$ & $<0.001$ \\
CFQ $^{\mathrm{a}}$ & $-0.364^{*}$ & 0.031 \\
\hline
\end{tabular}

a Pearson's r.

b Spearman's rho.

* Indicates correlation is significant at the 0.05 level (2-tailed).

** Indicates correlation is significant at the 0.01 level (2-tailed). correlation coefficients and significance levels found for RAI scores and all seven IQ indices and subindices. For the full correlational matrix, see Appendix B.

Regarding Verbal subtests, strong significant correlations were found between scores for the RAI and Arithmetic, Comprehension, Vocabulary, Information, with medium-strength correlations found for Similarities, Digit Span and Letter-Number Sequencing. In terms of Performance subtests, RAI scores displayed moderate significant correlation with scores for Block Design, Matrix Reasoning and Symbol Search, but did not significantly correlate with scores for Picture Completion, Digit-Symbol Coding or Picture Arrangement. Table 3 displays the correlation coefficients and significance levels for RAI correlations with each of these WAIS subtests.

In summary, Relational Ability Index scores showed significant moderate-to-strong correlations with the three main indices of IQ as well as the four IQ subindices. RAI scores also correlated significantly (with moderate-to-strong effects sizes) with 10 of 13 IQ subtests, indicating a considerable overlap with intellectual performance in general.

\section{General discussion}

The current analysis investigated the utility of a Relational Abilities Index as a potential proxy measure of intelligence, by assessing its degree of correlation with traditional IQ scores and a number of other IQ proxy measures. In Study 1, RAI scores were found to display a close relationship with scores on the NART, RAVLT and TMT. Importantly, the RAI scores diverged from scores on the CFQ, which does not itself predict IQ scores (Broadbent et al., 1982). In Study 2, RAI scores showed more than acceptable levels of correlation with Full Scale, Verbal and Performance IQ, each of the 4 IQ subindices, and 10 of 13 IQ subtests. These findings combine to suggest a high level of covariance between relational ability and IQ, supporting previous assertions regarding the 

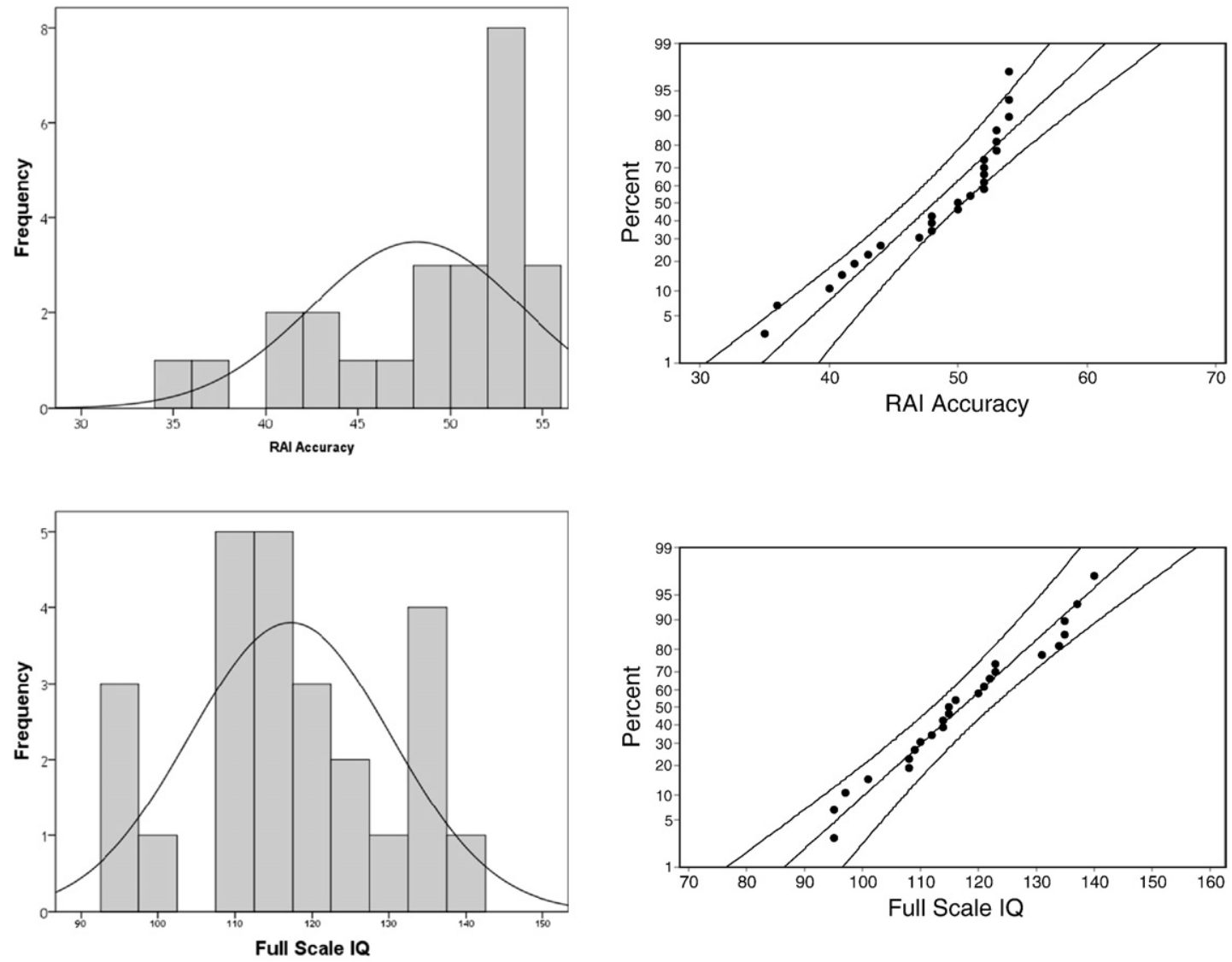

Fig. 3. Histograms (left) and probability plots (right) outlining the distribution of RAI scores and Full Scale IQ scores in Study 2.

central role of relational ability in intellectual performance (Andrews \& Halford, 1998; Barnes-Holmes, Barnes-Holmes, Stewart, \& Boles, 2010; Cattell, 1971; Dixon et al., 2014; Gentner \& Loewenstein, 2002; Gore et al., 2010; Halford, Wilson, \& Phillips, 2010; Moran et al., 2010; O'Hora et al., 2005, 2008; O'Toole et al., 2009; Stewart, Tarbox, Roche, \& O'Hora, 2013)

In assessing the validity of the RAI as a proxy measure, it is illuminating to compare the strength of correlation found between the RAI and WAIS Full Scale IQ scores $(r=0.74)$ to correlations found between Full Scale IQ and other established IQ proxy measures. The RAI-IQ correlation coefficients reported in the current study were similar to those found between scores for the WAIS-III and Ravens Standard Progressive Matrices (0.64; Wechsler, 1997), the Wechsler Individual Achievement Test (0.53-0.81; Silva, 2008), the Woodcock Johnson Test of Cognitive Ability (0.71; Cheramie, Stafford, Boysen, Moore, \& Prade, 2012; 0.82; Metz, 2005), the General Ability Measure for Adults (0.8; Martin, Donders, \& Thompson, 2000; 0.75; Naglieri \& Bardos, 1997), WASI

Table 2

Correlations between the RAI and WAIS III IQ index and IQ subindex scores.

\begin{tabular}{lll}
\hline IQ measure & Correlation coefficient & Significance level \\
\hline Full Scale IQ & $0.74^{* *}$ & $<0.001$ \\
Verbal IQ & $0.78^{* *}$ & $<0.001$ \\
Verbal Comprehension & $0.61^{* *}$ & 0.001 \\
Working Memory & $0.64^{* *}$ & 0.001 \\
Performance IQ & $0.55^{* *}$ & 0.004 \\
Perceptual Organisation & $0.53^{* *}$ & 0.006 \\
Processing Speed & $0.43^{*}$ & 0.03 \\
\hline
\end{tabular}

* Indicates correlation is significant at the 0.05 level (2-tailed).

** Indicates correlation is significant at the 0.01 level (2-tailed).
Verbal IQ (0.75; Axelrod, 2002) and the Oklahoma Premorbid Intelligence Estimate (0.69; Spinks et al., 2009).

The moderate-to-strong significant correlations between RAI scores and every WAIS Verbal subtest suggests that relational responding, at least as assessed by the RAI, is germane to linguistic performance and may possibly underlie language processes as suggested by a growing RFT literature. The results suggest that perhaps, for the current study at least, the strongest relationship between relational skills and aspects of intelligence lies in the verbal domain.

RAI scores correlated significantly with only three of six Performance IQ subtests. This suggests that the RAI may not tap into the same

Table 3

Correlations between the RAI and WAIS III subtest scores.

\begin{tabular}{lll}
\hline Verbal subtests & Correlation coefficient & Significance level \\
\hline Vocabulary & $0.63^{* *}$ & 0.001 \\
Similarities & $0.58^{* *}$ & 0.002 \\
Arithmetic & $0.75^{* *}$ & $<0.001$ \\
Digit Span & $0.52^{* *}$ & 0.007 \\
Information & $0.62^{* *}$ & 0.001 \\
Comprehension & $0.73^{* *}$ & $<0.001$ \\
Letter number sequencing & $0.5^{*}$ & 0.012 \\
Performance subtests & & \\
Picture completion & 0.02 & 0.92 \\
Digit symbol coding & 0.27 & 0.2 \\
Block design & $0.6^{* *}$ & 0.001 \\
Matrix reasoning & $0.48^{*}$ & 0.02 \\
Picture arrangement & 0.31 & 0.14 \\
Symbol search & $0.48^{*}$ & 0.02 \\
\hline
\end{tabular}

* Indicates correlation is significant at the 0.05 level (2-tailed).

** Indicates correlation is significant at the 0.01 level (2-tailed). 
intellectual skills repertoires involved in performance on standardized IQ tests, as much as it does index those skills recruited during verbal tasks. In addition, this finding emphasises that while the RAI may serve as a useful proxy or estimate of IQ it does not function as an alternate or short form assessment of IQ. Indeed, it is important to understand that a proxy IQ measure is merely an indirect measure of a standardized score, whose value is determined largely by the degree of correlation its scores bear to those produced by the standardized test. As such, an IQ proxy is a mere "indirect measure" of IQ (Spinks et al., 2009) and as such, need not necessarily have good face, or even construct validity (although this may be desirable). This is evinced by the common usage of IQ proxy measures, which vary markedly in form from traditional IQ assessments, such as Raven's Standard Progressive Matrices and the North American Adult Reading Test (NAART). The NAART, in particular, is a widely used proxy for IQ, but one that has low face and construct validity. This does not detract from its ability to provide indirect IQ estimates. As such, while the RAI appears to largely represent an assessment of verbal reasoning skills, this does not in any way take from the simple fact that its level of correlation with Full Scale IQ suggests that it may serve as a useful proxy.

Interestingly, the three WAIS subtests which did not correlate with RAI performance (Picture Completion, Digit-Symbol Coding \& Picture Arrangement) bear among the weakest relationships to Full Scale IQ along with Digit Span (Wechsler, 1997). Furthermore, in a comprehensive analysis of the respective $g$ loadings of Wechsler subtests, Gignac (2006) found that these three Performance subtests show the lowest $g$ loadings for the 13 subtests included in the current study. Of course, identifying divergences between relational skills and the skill battery assessed by traditional IQ tests is as important conceptually for the current research program as is the identification of convergences. However, all divergences are not equal conceptually, and it is important to note that the RAI scores correlated best precisely with those WAIS subtests that themselves have the highest $g$ loadings.

We are mindful that much remains to be understood regarding the relationship between relational responding and intelligence. In light of this, alternative and more aggressive analytic methods, such as multiple regression analysis, might better interrogate this relationship than did the correlational method used here. In the current study, however, our sample size was not sufficient to make such an analysis meaningful and it was in any case exploratory and was designed to provide the type of information and parameters that may inform such analyses in the future.

We are also aware, that in the absence of larger samples and more interrogating analysis, the correlations reported here can be interpreted in several different ways. For example, they may indicate that a high level of intelligence is required to perform well on relational tasks, rather than vice versa. Alternatively, they may point to a third variable that accounts for performance on both the WAIS and the RAI. Indeed, such a possibility exists even where controlled gains in relational skill have led to increases in IQ.

The RAI would appear to offer a number of advantages over conventional IQ and IQ-proxy assessments. Firstly, it is a relatively short test, easily administered, even without supervision, using web-based technology. Secondly, the RAI contains culture-free test items. Thirdly, every administration constitutes an alternate form, which reduces practice effects and may explain the RAI's respectable test-retest reliability. Fourthly, the RAI may be of use to behavioral researchers or psychometricians who do not subscribe to the existence of $g$. Such researchers view intelligence in terms of more fluid skills repertoires and may appreciate a test that also measures the repertoire from this perspective. Moreover, as the nature of the skills assessed by the RAI are now fairly well understood from hundreds of published studies into DRR, the meaning of RAI scores (i.e., an index of DRR proficiency) is easily interpreted, free from debate regarding hypothetical constructs that the RAI aims to measure.
It is important to remember that the current RAI test was a firstbuild based entirely on conceptual principles, rather than backward engineered through psychometric statistical analyses. The four relational skills assessed in the current build (Same, Opposite, More and Less) were chosen because these relations appear to be assessed heavily in IQ tests (see Cassidy et al., 2010; Stewart et al., 2013) and are fundamental to language acquisition and logical reasoning (see Hayes et al., 2001). However, many IQ test items tap into more complex relational skills, and failures of the RAI to correlate well with certain subtests of the WAIS may relate to the limited scope of the current RAI (see Gore et al., 2010; McHugh, Barnes-Holmes, \& Barnes-Holmes, 2004; O'Hora et al., 2008). In light of this, it may be beneficial to include a wider range of relational frame assessment tasks in future versions of the RAI in order to more fully describe and index the entire skills repertoire currently assessed by IQ tests.

RAI scores were not normally distributed for the sample of participants recruited for Study 2. The concentration of scores towards the upper limit of the RAI maximum surely compromised the degree of correlations reported here. Interestingly, however, respectable correlations between RAI scores and IQ scores were obtained even despite this slight skew in the distributions of obtained scores and more impressive correlations would be expected if a more representative sample were to be employed in future studies.

One important modification to the RAI might involve a restructuring of its scoring system to reflect the increasingly challenging nature of trials across the test block. As the scoring system employed by the RAI is a summated scale, it does not reflect the difference in value of responses early or late in the test block. A possible alternative system would be a Guttman-style scale, that would arrange test items in a cumulative order (e.g. order of difficulty) allowing the assumption that if an individual correctly responds to a given test item, he/she will also correctly respond to previous test items (Guttman, 1954; Manheim, 1977; Mokken, 1970). This restructuring of the scaling system employed by the RAI may therefore render the resulting score more sensitive to individual differences.

It may also be prudent to compute a fluency score, which would take into account not only accuracy, but also speed of responding. Due to the functional importance of speed in learning tasks within the behavioral tradition (Binder, 1996), this may in fact improve the utility of the RAI in assessing relational ability as well as intellectual performance. This will involve further investigations into the relationship between speed of relational responding and various task performances considered important in a behavior-analytic conceptualization of intelligence. What will not be acceptable, from a functional-analytic perspective, however, is to adjust the speed weighting in a fluency measure, in order to achieve the highest possible correlation between RAI fluency scores and IQ. Such a psychometric strategy would represent the backward engineering of a new IQ test. It would also leave IQ unexamined functionally and would assume any divergences between IQ and RAI scores to be based entirely on deficiencies in the construct validity of the RAI measure. Such a research strategy could therefore yield little of interest to the literature on intelligence testing.

\section{Conclusion}

The results of the current analyses offer preliminary support for the Relational Abilities Index as a potential proxy measure of intelligence. The level of correlation found between Full Scale IQ and RAI scores would appear to confirm the RAI's status as an efficacious means of describing intellectual performance, and is at a level at least comparable to those reported for several widely accepted proxy and short-form measures of intellectual performance (e., Raven's Matrices; Silva, 2008). As such, the RAI may represent a promising new proxy measure of IQ for those who adopt a functional approach to the assessment of intellectual skills. 
Appendix A

Table A1

Full Correlation Matrix for RAI scores and cognitive abilities battery.

\begin{tabular}{|c|c|c|c|c|c|}
\hline Measure & 1 & 2 & 3 & 4 & 5 \\
\hline 1. RAI & - & & & & \\
\hline 2. NART & $0.58^{* *}, \mathrm{a}$ & - & & & \\
\hline 3. RAVLT 1 to 5 & $0.7^{* *}, \mathrm{a}$ & $-0.51^{*}, \mathrm{a}$ & - & & \\
\hline 4. TMT B-A & $-0.36^{*}, \mathrm{~b}$ & $.26^{\mathrm{b}}$ & $-0.4^{*}, \mathrm{~b}$ & - & \\
\hline 5. CFQ & $-.08^{\mathrm{a}}$ & 0.03 & $-.02^{\mathrm{a}}$ & $.12^{\mathrm{b}}$ & - \\
\hline
\end{tabular}

* Indicates correlation is significant at the 0.05 level (2-tailed).

** Indicates correlation is significant at the 0.01 level (2-tailed).

a Pearson's $\mathrm{r}$.

b Spearman's rho.

\section{Appendix B}

Table B1

Full Correlation Matrix for RAI scores and IQ indices and subindices.

\begin{tabular}{|c|c|c|c|c|c|c|c|c|}
\hline Measure & 1 & 2 & 3 & 4 & 5 & 6 & 7 & 8 \\
\hline 1. RAI & - & & & & & & & \\
\hline 2. Full Scale IQ & $0.739^{* *}$ & - & & & & & & \\
\hline 3. Verbal IQ & $0.781^{* *}$ & $0.933^{* *}$ & - & & & & & \\
\hline 4. Performance IQ & $0.554^{* *}$ & $0.866^{* *}$ & $0.635^{* *}$ & - & & & & \\
\hline 5. Verbal comprehension & $0.608^{* *}$ & $0.827^{* *}$ & $0.869^{* *}$ & $0.582^{* *}$ & - & & & \\
\hline 6. Perceptual organisation & $0.531^{* *}$ & $0.835^{* *}$ & $0.645^{* *}$ & $0.924^{* *}$ & $0.589^{* *}$ & - & & \\
\hline 7. Working memory & $0.644^{* *}$ & $0.839^{* *}$ & $0.866^{* *}$ & $0.630^{* *}$ & $0.629^{* *}$ & $0.626^{* *}$ & - & \\
\hline 8. Processing speed & $0.426^{*}$ & 0.341 & 0.224 & $0.445^{*}$ & 0.140 & 0.234 & 0.286 & - \\
\hline
\end{tabular}

* Indicates correlation is significant at the 0.05 level (2-tailed).

** Indicates correlation is significant at the 0.01 level (2-tailed).

\section{References}

Abramson, C. I. (2013). Problems of teaching the behaviorist perspective in the cognitive revolution. Behavioral Science, 3(1), 55-71 http://doi.org/10.3390/bs3010055.

Andrews, G., \& Halford, G. S. (1998). Children's ability to make transitive inferences: The importance of premise integration and structural complexity. Cognitive Development 13, 479-513.

Ardila, A., Pineda, D., \& Rosselli, M. (2000). Correlation between intelligence test scores and executive function measures. Archives of Clinical Neuropsychology, 15(1), 31-36.

Axelrod, B. N. (2002). Validity of the Wechsler Abbreviated Scale of Intelligence and other very short forms of estimating intellectual functioning. Assessment, 9, 17-23.

Barnes-Holmes, Y., Barnes-Holmes, D., Roche, B., \& Smeets, P. (2001). The development of self and perspective-taking: A relational frame analysis. Behavioral Development Bulletin, 10(1), 42-45http://dx.doi.org/10.1037/h0100482

Barnes-Holmes, D., Barnes-Holmes, Y., Stewart, I., \& Boles, S. (2010). A sketch of the implicit relational assessment procedure (IRAP) and the relational elaboration and coherence (REC) model. Psychological Record, 60, 527-542.

Binder, C. (1996). Behavioral fluency: Evolution of a new paradigm. The Behavior Analyst $19,163-197$.

Block, N. (1981). Psychologism and behaviorism. Philosophical Review, XC(1), 5-43.

Bolla-Wilson, K., \& Bleecker, M. L. (1986). Influence of verbal intelligence, sex, age, and education on the Rey Auditory Verbal Learning Test. Developmental Neuropsychology, 2 203-211.

Broadbent, D. E., Cooper, P. F., Fitzgerald, P., \& Parkes, K. R. (1982). The cognitive failures questionnaire and its correlates. British Journal of Clinical Psychology, 21, 1-16.

Cassidy, S., Roche, B., Colbert, D., Stewart, I., \& Grey, I. (2016). A relational frame skills training intervention to increase general intelligence and scholastic aptitude. Learning and Individual Differences, 47, 222-235.

Cassidy, S., Roche, B., \& Hayes, S. C. (2011). A relational frame training intervention to raise Intelligence Quotients: A pilot study. The Psychological Record, 61, 173-198.

Cassidy, S., Roche, B., \& O'Hora, D. (2010). Relational Frame Theory and human intelligence. European Journal of Behavior Analysis, 11, 37-51.

Cattell, R. (1971). Abilities. Boston: Houghton Mifflin.

Cheramie, G. M., Stafford, M. E., Boysen, C., Moore, J., \& Prade, C. (2012). Relationship between the Wechsler Adult Intelligence Scale - Fourth Edition (WAIS-IV) and Woodcock-Johnson-III Normative Update (NU): Tests of Cognitive Abilities (WJ-III: COG). Journal of Education and Human Development, 5(1), 1-9.

Crawford, J. R., Parker, D. M., Stewart, S. E., Besson, J. A. O., \& De Lacey, G. (1989a). Prediction of WAIS IQ with the National Adult Reading Test: Cross-validation and extension. British Journal of Clinical Psychology, 28, 267-273.

Crawford, J. R., Stewart, L. E., Cochrane, R., Parker, D. M., \& Besson, J. A. O. (1989b). Construct validity of the National Adult Reading Test: A factor analytic study. Personality and Individual Differences, 10, 585-587.

Crossen, J. R., \& Wiens, A. N. (1994). Comparison of the auditory verbal learning test (AVLT) and California Verbal Learning Test (CVLT) in a sample of normal subjects. Journal of Clinical and Experimental Neuropsychology, 16(2), 190-194.
Dixon, M., Whiting, S., Rowsey, K., \& Belisly, J. (2014). Assessing the relationship between intelligence and the PEAK relational training system. Research In Autism Spectrum Disorders, 8(9), 1208-1213http://dx.doi.org/10.1016/j.rasd.2014.05.005

Dymond, S., \& Roche, B. (2013). Advances in relational frame theory. Oakland, CA: Context Press.

Esher, F., Raven, I., \& Earl, C. (1942). Discussion on testing intellectual capacity in adults. Proceedings of the Royal Society of Medicine, 35, 779-785.

Gentner, D., \& Loewenstein, J. (2002). Relational language and relational thought. In J. Byrnes, \& E. Amsel (Eds.), Language, literacy, and cognitive development (pp. 87-120). Mahwah, NJ: LEA

Gignac, G. E. (2006). Evaluating the subtest ' $g$ ' saturation level via the single trait-correlated uniqueness (STCU) SEM approach: Evidence in favor of crystallized subtests as the best indicators of ' $\mathrm{g}$ '. Intelligence, 34, 29-46.

Gore, N. J., Barnes-Holmes, Y., \& Murphy, G. (2010). The relationship between intellectual functioning and relational perspective-taking. International Journal of Psychology and Psychological Therapy, 10, 1-17.

Gottfredson, L. (1998). The general intelligence factor. New York: Scientific American, Inc. Gould, S. (1981). The mismeasure of man. New York: Norton.

Guttman, L. (1954). An outline of some new methodology for social research. Public Opinion Quarterly, 18(4), 395-404http://dx.doi.org/10.1086/266532

Halford, G., Wilson, W., \& Phillips, S. (2010). Relational knowledge: The foundation of higher cognition. Trends in Cognitive Sciences, 14(11), 497-505.

Hayes, S. C. (1994). Relational frame theory as a behavioral approach to verbal events. In S. C. Hayes, L. J. Hayes, M. Sato, \& K. Ono (Eds.), Behavior analysis of language and cognition (pp. 9-30). Reno, NV: Context Press.

Hayes, S., Barnes-Holmes, D., \& Roche, B. (2001). Relational frame theory. New York: Kluwer Academic/Plenum Publishers.

Hayes, J., \& Stewart, I. (2016). Comparing the effects of derived relational training and computer coding on intellectual potential in school-age children. British Journal of Educational Psychology, 86, 397-411. http://dx.doi.org/10.1111/bjep.12114.

Heaton, R. K., Grant, I., \& Matthews, C. G. (1986). Differences in neuropsychological test performance associated with age, education and sex. In I. Grant, \& K. M. Adams (Eds.), Neuropsychological assessment of neuropsychiatry disorders (pp. 100-120). New York: Oxford University Press.

Herrmann, D. J. (1982). Know thy memory: The use of questionnaires to assess and study memory. Psychological Bulletin, 92, 434-452.

Howe, M. (1990). The origins of exceptional abilities. Oxford: Blackwell.

Kaufman, A. S., \& Kaufman, N. L. (2004). Kaufman Brief Intelligence Test (2nd ed.). Bloomington, MN: Pearson.

Lezak, M. D. (1995). Neuropsychological assessment (3rd ed.). New York: Oxford University Press.

Manheim, H. L. (1977). Sociological research. Georgetown: Irwin Dorsey.

Martin, M. (1986). Aging and patterns of change in everyday memory and cognition. Human Learning, 5, 63-74.

Martin, T. A., Donders, J., \& Thompson, E. (2000). Potential of and problems with new measures of psychometric intelligence after traumatic brain injury. Rehabilitation Psychology, 45, 402-408. 
Martin, M., \& Jones, G. V. (1983). Distribution of attention in cognitive failure. Human Learning, 2, 221-226.

McHugh, L., Barnes-Holmes, Y., \& Barnes-Holmes, D. (2004). Perspective-taking as relational responding: A developmental profile. The Psychological Record, 54, $115-144$.

Metz, B. L. (2005). A comparison of the WJ-III Test of Cognitive abilities and the WAIS-III. Theses, Dissertations and Capstone762.

Mitrushina, M., Boone, K. B., Razani, J., \& D'Elia, L. F. (2005). Handbook of normative data for neuropsychological assessment (2nd ed.). New York: Oxford University Press.

Mokken, R. J. (1970). A theory and procedures of scale analysis: With applications in political research. The Hague: Mouton.

Moran, L., Stewart, I., McElwee, J., \& Ming, S. (2010). Brief Report: The Training and Assessment of Relational Precursors and Abilities (TARPA): A preliminary analysis. Journal of Autism and Developmental Disorders, 40(9), 1149-1153.

Naglieri, J. A., \& Bardos, A. N. (1997). General Ability Scale for Adults (GAMA). Minnetonka, NM: National Computer Systems.

Nelson, H. (1982). National Adult Reading Test (NART): Test manual. Windsor, U.K.: NferNelson.

O'Hora, D., Pelaez, M., \& Barnes-Holmes, D. (2005). Derived relational responding and performance on verbal subtests of the WAIS-III. The Psychological Record, 55, 155-175.

O'Hora, D., Pelaez, M., Barnes-Holmes, D., Rae, G., Robinson, T., \& Chaudhary, T. (2008). Temporal relations and intelligence: Correlating relational performance with performance on the WAIS-III. The Psychological Record, 58, 569-583.

O'Toole, C., Barnes-Holmes, D., Murphy, C., O'Connor, J., \& Barnes-Holmes, Y. (2009). Relational flexibility and intelligence: Extending the remit of Skinner's Verbal Behavior. International Journal of Psychology and Psychological Therapy, 9, 1-17.

Putnam, H. (1975). Mind, language and reality, philosophical papers. vol. 2.Cambridge: Cambridge University Press.
Rey, A. (1958). L'examen clinique en psychologie. Paris: Presses Universitaire de France. Schlinger, H. D. (2003). The myth of intelligence. The Psychological Record, 53(1), 15-33. Silva, M. A. (2008). Development of the WAIS-III: A brief overview, history and description. Graduate Journal of Counseling Psychology, 1(1), 1-19.

Skinner, B. F. (1974). About behaviorism. New York: Alfred A. Knopf.

Smith, B., Smith, T., Taylor, L., \& Hobby, M. (2005). Relationship between intelligence and vocabulary 1,2. Perceptual and Motor Skills, 100(1), 101-108http://dx.doi.org/10. 2466/pms.100.1.101-108

Spinks, R., McKirgan, L. W., Arndt, S., Caspers, K., Yucuis, R., \& Pfalzgraf, C. J. (2009). IQ estimate smackdown: Comparing IQ proxy measures to the WAIS-III. Journal of the International Neuropsychological Society, 15(4), 590-596.

Spreen, O., \& Strauss, E. (1998). A compendium of neuropsychological tests: Administration, norms, and commentary (2nd ed.). New York: Oxford University Press.

Sternberg, R. (1977). Intelligence, information processing, and analogical reasoning. Hillsdale, N.J: Lawrence Erlbaum Associates.

Stewart, I., Tarbox, J., Roche, B., \& O'Hora, D. (2013). Education, intellectual development, and relational frame theory. In S. Dymond, \& B. Roche (Eds.), Advances in relationa frame theory: Research \& application (pp. 178-198). Oakland, CA: New Harbinger.

Taylor, E. M. (1959). The appraisal of children with cerebral deficits. Cambridge, MA: Harvard University Press.

Wechsler, D. (1955). Wechsler Adult Intelligence Scale Manual. New York: Psychological Corporation.

Wechsler, D. (1997). WAIS-III WMS-III technical manual. San Antonio, TX: The Psychological Corporation.

Wechsler, D. (1999). Wechsler Abbreviated Scale of Intelligence manual. San Antonio, TX: Psychological Corporation. 